\title{
Les marchés financiers sont-ils politiquement corrects ?
}

par Sebastián Nieto Parra et Javier Santiso, Centre de développement de I'OCDE

- Les recommandations des banques d'investissement concernant les emprunts publics des pays émergents tendent à devenir particulièrement défavorables à l'approche d'une élection.

- Cette aversion est en fait davantage liée à l'incertitude que génère l'évènement qu'à la nature même de celui-ci. En particulier, les programmes déclarés par les candidats, notamment en matière monétaire et budgétaire, sont cruciaux pour la stabilité des recommandations des banques pendant les périodes électorales.

Les marchés financiers sont-ils politiquement corrects ? Autrement dit ont-ils des préférences en matière de régimes politiques ou une partisane ? Cette question a souvent été explorée pour les investissements directs étrangers (IDE) ou encore l'aide publique au développement, mais rarement pour les flux de portefeuille dans les pays émergents.

L'univers où investissent les gérants de portefeuilles d'actions et d'obligations dans la sphère des marchés émergents est relativement étroit. Il se limite généralement aux indices MSCI (Morgan Stanley Capital International) pour les investissements en actions, et EMBI (Emerging Markets Bond Index) pour les investissements obligataires. Le nombre des pays référenciés par ces indices oscille entre 30 et 40 . Tous, à deux ou trois exceptions près, sont des démocraties. On pourrait en conclure que les marchés financiers s'orientent massivement en faveur des régimes démocratiques. Ils seraient donc, à première vue, « politiquement corrects ».

Cependant, en y regardant de plus près, ce premier constat se lézarde. En effet, la plupart des grandes crises financières qui ont secoué les pays émergents ont coïncidé avec des élections. C'est vrai par exemple pour l'Amérique latine, où toutes les grandes crises sont intervenues lors de scrutins présidentiels, comme au Mexique en 1994, et au Brésil en 1999 (quelques mois après l'élection d'octobre 1998) puis à nouveau en 2002. Autrement dit, les marchés financiers tendent à devenir particulièrement défavorables à l'approche d'une élection.

Cette aversion est en fait davantage liée à l'incertitude que génère l'évènement qu'à la nature même de celui-ci.
Son intensité varie en fonction de la probabilité de voir certains candidats l'emporter (en général, les candidats situés à gauche du spectre politique induisent davantage d'aversion). Ce phénomène n'est pas statique mais dynamique, il varie dans le temps. Telles sont certaines des conclusions d'une étude empirique inédite menée récemment sur les marches émergents ${ }^{1}$.

À partir d'une base de données unique couvrant plus de 10 ans (1997-2008) et contenant plus de 5000 observations, on a exploré comment réagissent les analystes financiers en périodes électorales dans les démocraties émergentes. Au total près de 700 rapports financiers ont été décortiqués provenant de 13 banques d'affaires. L'analyse se concentre sur les marchés émergents obligataires et sur ceux d'Amérique latine, mais elle pourrait être répliquée pour l'ensemble des pays émergents, et étendue aux investissements en actions.

On a étudié l'ensemble des recommandations, positives ou négatives, de ces analystes à l'approche du scrutin. De manière générale, les banques tendent à mettre sous surveillance le pays concerné pendant les trois mois qui précèdent l'élection, ou à réduire leur biais, en général positif. Les recommandations des banques sont particulièrement sensibles à la crédibilité qu'elles portent aux programmes déclarés par les candidats, notamment en matière monétaire et budgétaire.

1. Voir Sebastián Nieto Parra et Javier Santiso, "Wall Street and Elections in Latin American Emerging Democracies", Working Paper No. 272, Centre de développement de I'OCDE, 2008. Ce travail a été présenté à LACEA 2007, à la Banque Centrale d'Espagne (Banco de España) et à la Banque des Règlements Internationaux (BRI). 
Cette sensibilité au cycle électoral n'est cependant pas uniforme. Pour certains pays émergents comme le Chili, par exemple, les élections n'ont pratiquement plus d'impact. Ce type de pays reste cependant l'exception, mais on constate qu'en 2006, l'intensité du cycle politique n'a pas suscité de phénomènes d'aversions comparables à ceux enregistrés lors de cycles précédents.

Les analystes de banques s'avèrent ainsi particulièrement sensibles aux signaux envoyés par les candidats présidentiels. Ils jugent et jaugent de manière détaillée leurs promesses et ajustent leurs recommandations en fonction. Quand les candidats paraissant en mesure de l'emporter alignent des promesses considérées peu crédibles ou de nature à affecter l'équilibre macroéconomique du pays, les analystes invitent leurs clients, les gérants de portefeuilles et autres investisseurs, à réduire leurs investissements.

Les gouvernants, s'ils souhaitent être réélus ou aspirent à se représenter, ont tous un intérêt convergeant à ne pas assumer le pouvoir dans des conditions de crise économique. Dans certains cas, comme par exemple en 2001 au Brésil2, les sortants peuvent ajuster leurspromesses et parfois même se lier les mains (avec le FMI par exemple), donnant ainsi un signal fort en direction des marchés quant à la crédibilité de leurs programmes.

2. Pour plus de détails voir Juan Martínez et Javier Santiso, "Financia Markets and Politics: The Confidence Game in Latin American Emerging Democracies", International Political Science Review, 2003, vol. 24 (39) pp. 363-395.
L'exemple du Brésil est également intéressant en matière de stratégie préventive: en 2001, année électorale, les autorités en place avaient délibérément réduit les volumes de dette arrivant à échéance, en prévision de possibles turbulences. Au final, la stratégie s'est avérée non seulement sage, car les marchés ont bel et bien sur-réagi, effrayés de voir un candidat de gauche (en I'occurrence Lula) arriver au pouvoir, mais elle aura permis sans doute d'éviter le pire. Par la suite, les ajustements et réajustements de programmes du président Lula ont montré que ces anticipations négatives étaient infondées, et les marchés ont vécu ensuite une véritable « Lula de mel » (allusion à « lune de miel ») avec le nouveau gouvernement brésilien.

De telles stratégies préventives ou d'ancrage international sont des réponses possibles à l'aversion des marchés financiers face aux incertitudes de toute élection. Elles sont d'autant plus bienvenues que, comme on l'a souligné, les marchés sont loin d'être indifférents aux évènements politiques. Bien au contraire, une élection dans un pays émergent est une échéance tenue pour décisive par Wall Street. Cette sensibilité des marchés au cycle politique est d'ailleurs une caractéristique des pays émergents.
Les lecteurs sont invités à citer ou reproduire les informations des Repères du Centre de développement de I'OCDE dans leurs propres publications. En échange, le Centre demande les remerciements de rigueur ainsi qu'un exemplaire de la publication. Le texte intégral des Repères et d'autres informations sur le Centre de développement et ses travaux sont disponibles sur : www.oecd.org/dev
Centre de développement de I'OCDE 2 , rue André-Pascal, 75775 Paris Cedex 16, France Tél : $33(0) 145.24 .82 .00$

Fax : $33(0) 144.30 .61 .49$ mél : cendev.contact@oecd.org 